Euskal ikerketen aldizkaria | Revue d'études basques |

Revista de estudios vascos | Basque studies review

$5 \mid 2000$

Numéro $\mathrm{V}$

\title{
Gernikako Arbola véritable hymne basque
}

Jean Haritschelhar

\section{OpenEdition \\ Journals}

Édition électronique

URL : http://journals.openedition.org/lapurdum/1332

DOI : 10.4000/lapurdum.1332

ISSN : 1965-0655

Éditeur

IKER

Édition imprimée

Date de publication : 1 octobre 2000

Pagination : 207-322

ISBN : 2-84127-161-7

ISSN : $1273-3830$

\section{Référence électronique}

Jean Haritschelhar, «Gernikako Arbola véritable hymne basque », Lapurdum [En ligne], 5 | 2000, mis en ligne le 01 juin 2009, consulté le 02 juillet 2020. URL : http://journals.openedition.org/lapurdum/1332 DOI : https://doi.org/10.4000/lapurdum.1332

Ce document a été généré automatiquement le 2 juillet 2020

Haritschelhar J. | IKER 


\title{
Gernikako Arbola véritable hymne basque
}

\author{
Jean Haritschelhar
}

1 Il n'existe pas une grande différence entre le bertsulari ou improvisateur et l'auteur de bertsu berri. Certes, le premier, comme son nom ne l'indique pas en basque, improvise ses couplets alors que l'autre prend tout son temps, peut, à tout moment, apporter une correction avant que son œuvre ne soit livrée au public, mais ils ont en commun une même technique c'est à dire que, de même que l'improvisateur compose sur un air donné, l'auteur de « chansons nouvelles » utilise aussi une musique connue pour écrire ses paroles. Il se peut que le bertsulari se transforme en auteur de chansons, alors que l'inverse n'est pas vrai. Manuel Lekuona va jusqu'à penser que certains improvisateurs ne possédant pas une belle voix sont devenus auteurs de bertso berri en confiant ainsi à d'autres le soin de chanter leur production :

«La circunstancia de una voz poco potente o cualquiera otra de carácter más o menos personal ha hecho que muchos de los bertsolaris no contraigan el hábito de exhibirse en público como otros de sus compañeros - llamados por esto plaza gizon - mejor dotados que ellos de facultades de expresión y presentación ${ }^{1}$. "

D'autres, par contre, ne craignant pas de se montrer en public dans des joutes entre improvisateurs, ont aussi de la même manière composé des chansons qu'ils ont adressées au jury des concours d'Abbadie comme par exemple Pierre Ibarrart ou encore Jean-Baptiste Oxalde tous deux lauréats des Jeux floraux, le premier interdit d'improvisation en public par le curé de Baigorri depuis qu'il était chantre en l'église alors que l'autre participait à toutes les fêtes, toberak ou autres. Cette technique est parfaitement illustrée par l'incipit de nombreuses chansons tel que :

Ahaire zahar huntan bi berset berririk

Alagrantziareki khantatü nahi tit.

chanson souletine anonyme ${ }^{2}$.

La formule a des variantes, mais s'en remet toujours à l'opposition entre zahar et berri

Airea zahar eta kantorea berri

Sujetak ere dire anhitz xarmagarri ${ }^{3}$ 
que le poète saratar Elissamburu n'hésitera pas à employer dans l'une de ses productions.

Mila bat zortzi ehun eta lau etan hogoiean

Bertsu berri hauk eman dire aire zahar batean ${ }^{4}$

5 Ainsi, pendant des siècles - les chansons basques les plus anciennes datent du XIV ${ }^{e}$ siècle - s'est perpétuée une tradition que les Jeux floraux organisés par Antoine d'Abbadie et ses amis n'allaient pas rompre, ainsi que le marque l'appel pour le premier concours de poésie qui eut lieu en 1853.

«Quelques amis du Pays Basque nous prient d'annoncer qu'ils donneront le 5 septembre prochain sur la place d'Urrugne et avant la partie de balle, une once d'or et un makila ou bâton basque à l'auteur de la meilleure chanson exprimant les regrets d'un Basque en partance pour Montevideo.

La chanson ne devra pas avoir plus de cinquante vers, être en strophes, et être adaptée sur un air basque connu que l'auteur indiquera ${ }^{5}$. »

7

81853 : la date est de celles qui comptent dans l'histoire de la littérature basque. En effet, comme je viens de le dire, est organisé le premier concours de poésie basque au cours des Jeux floraux qui dureront jusqu'en 1914 et, à Madrid, au café San Luis de la calle de la Montera, José Maria Iparraguirre crée le Gernikako arbola. Deux événements importants, le premier ancré dans la tradition et la ruralité puisqu'il se déroule à Urrugne, l'autre, au contraire, ayant lieu dans la capitale espagnole, œuvre d'un auteur-compositeur, interprète brillant, accompagné au piano par Juan Maria Blas Altuna.

Deux mondes s'opposent, le monde baserritar (rural) et le monde kaletar (citadin), celui de la création poétique dépendant d'une tradition musicale et celui de la création totale, paroles et musique.

10 Cette opposition est symbolisée par le couplet improvisé par le bertsulari Xenpelar, toujours fidèle à la tradition et n'appréciant guère le comportement d'Iparraguirre.

Iparraguirre abilla dela

askorï diyot aditzen...

Eskola ona eta musika

ori oyekin serbitzen.

Ni enazu ibiltzen

kantuz dirua biltzen

komeriante moduan ;

debalde pesta preparatzen det

gogua dedan orduan ${ }^{6}$.

La critique est sévère et Xenpelar n'y va pas de main morte. Le citadin est décrit comme rusé (abilla), sachant y faire, non seulement lettré (eskola ona), mais en outre musicien, sachant se servir d'une guitare puisque c'est ainsi que se présente Iparraguirre (oyekin serbitzen). En face, le baserritar, Ni, Xenpelar lui-même qui, au contraire, ne s'exhibe jamais à la manière d'un histrion (komeriante moduan) battant les estrades pour gagner de l'argent (kantuz dirua biltzen). Xenpelar, en revanche, sait faire la fête quand il lui plaît (gogua dedan orduan) et surtout gratuitement (debalde). En vérité, Iparraguirre se présente dans le monde basque comme un être à part, bousculant les habitudes et les 
traditions. Il est vrai que sa destinée est singulière et quasiment digne de légende ; il est et sera le romantique basque par excellence.

Né en 1820 à Villarreal de Urretxu dans une famille de commerçants, il suivra ses parents à Madrid et un jour de 1834 - il a donc 14 ans - il dit à sa mère qu'il va à l'école et ne rentre plus chez lui. Il rejoint le Pays Basque et s'engage dans les troupes carlistes malgré son jeune âge. Il combat, il est blessé et finalement il fera partie de la garde d'honneur du roi Don Carlos V Refusant le pacte de Vergara, il s'exile en France où il apprend, outre le français, le chant et la musique. Doué d'une belle voix de baryton, il se produira sur diverses scènes, en France, en Italie, en Angleterre, recueillant ainsi le qualificatif de arlote, c'est à dire vagabond.

13 Il est, en fait, le premier auteur-compositeur interprète connu en Pays Basque. À coup sûr il dérange et en particulier le cercle des bertsularis. C'est pourquoi Xenpelar lui lancera le défi fameux dans les annales.

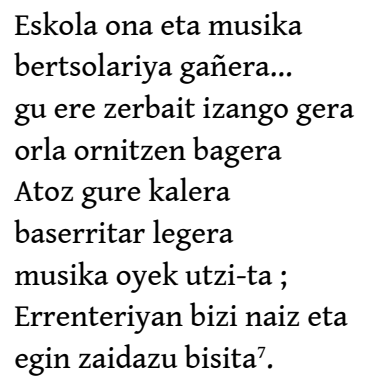

14 Xenpelar enchaîne un deuxième couplet en reprenant ce qu'il avait évoqué au précédent : instruit et musicien (eskola ona eta musika), et de surcroît improvisateur (bertsolariya gañera), ce qui complète le portrait.

Vient ensuite le défi en bonne et due forme, les conditions étant posées : baserritar legera, c'est à dire de manière rurale, traditionnelle en laissant de côté tout l'appareil musical (musika oyek utzi-ta). En fait, on assiste au rituel du défi, connu depuis l'antiquité: injures lancées à l'ennemi, conditions posées, lieu de la rencontre (Errenteriyan bizi naiz), date à la convenance du défié (Atoz gure kalera... egin zaidazu bisita).

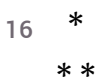

17 1853: il y a quatorze ans que la première guerre carliste s'est terminée par le « convenio de Vergara» que J. M. Iparraguirre n'a pas accepté. C'est dire son engagement carliste et surtout « fueriste ».

Depuis Vergara l'inquiétude règne dans les provinces basques car la question des « fueros » est posée. La loi du 25 octobre 1839, conséquence de l'article ${ }^{\mathrm{er}}$ du Convenio de Vergara, confirme, certes, les fueros des "Provincias Vascongadas y de Navarra ", mais la seconde partie du même article ajoute: «sin perjuicio de la unidad constitucional de la Monarquía ». D'autre part, l'article 2 de la même loi stipule que, après avoir entendu les délégués des provinces basques, le gouvernement proposera aux "Cortes » la modification des fueros. Les délégués furent nommés en 1840, mais ni en 1844, ni en 1846 et non plus en 1852 on ne parvint à un accord. Federico de Zabala dans son article intitulé « Iparraguirre y su entorno Los fueros » marque bien l'ambiance de l'époque en Pays Basque. 
« Un malestar, un descontento, una inquietud, una inestabilidad quizás algo vaga y difusa, unido todo ello al recuerdo de su felicidad bajo el régimen forai, hizo que la adhesión, el amor a los fueros se hiciera más vivo y mas fuerte ${ }^{8}$. "

Et il ajoute quelques lignes plus loin :

« En esta situación, en estas circunstancias llega a Bilbao Iparraguirre. Al pueblo le faltaba una voz que fuera la intérprete de los sentimientos más nobles ; faltaba esa voz musical semejante al ritmo del corazón de todo un pueblo, y eso fue Iparraguirre. Fue el poeta precursor del despertar vasco ${ }^{9}$.

C'est effectivement dans ce contexte particulier que J. M. Iparraguirre revient de son exil de Londres grâce au général Mazarredo qui l'a entendu chanter et qui intercède en sa faveur. A son retour, il se rend compte de l'inquiétude qui règne en Pays Basque au sujet des fueros, ces fueros que le gouvernement veut modifier par l'intermédiaire du Parlement, alors que, depuis quelques siècles, c'est le Roi qui vient jurer qu'il les respectera. Pour seule riposte, le poète utilisera ses propres armes, le poème chanté à la gloire de l'arbre de Guernica, symbole des libertés basques. Le poème est composé de douze strophes de quatre vers (zortziko txiki), mais on ne peut assurer que cette version de douze strophes fut celle qui fut chantée en 1853 au café San Luis.

21 En effet, on se trouve en présence de diverses versions, certaines de quatre strophes, d'autres de huit et d'autres encore de douze. Il semblerait que celles de quatre soient les plus anciennes.

Au sujet de celles de douze strophes, Manterola est catégorique dans son jugement :

«Chaho, Michel, Trueba y otros escritores sólo orrecen cuatro únicas estrofas... la edición hecha por el señor Santesteban consta a su vez hasta de doce estrofas. Por mi parte he aceptado esta última versión, que es la más conocida, pero... suprimiendo sus cuatro últimas estrofas que nada añaden de esencial a la composición y que en cambio perjudican, en mi sentir, de una manera notable a su robustez y vigor, haciéndola un tanto pesada y monótona ${ }^{10}$. »

Il semble donc que Manterola, même s'il accepte la version de douze strophes, pencherait plutôt pour celle de huit qui lui apparaît former une unité véritable. Toutefois je rejoindrai l'opinion de Juan Mari Lekuona qui voit dans l'ensemble de douze strophes trois mouvements parfaitement marqués de quatre strophes, chacun se terminant d'ailleurs par une prière.

24 Le thème de l'arbre de Guernica a été fort judicieusement choisi par J. M. Iparraguirre. L'arbre de Guernica n'est pas simplement connu des Biscayens ou des Basques. Son renom est tel que Tirso de Molina y fait allusion et que Jean-Jacques Rousseau en fait le symbole des libertés basques.

D'autre part, dans une vision «fueriste », Guernica est l'endroit où les Rois de Castille venaient jurer leur attachement aux fueros biscayens. Étant donné l'époque où règne l'inquiétude et toute la symbolique liée à l'arbre, et au chêne en particulier, l'exaltation du chêne de Guernica recueillera l'adhésion populaire.

En effet le Gernikako arbola doit être essentiellement analysé à travers la symbolique de l'arbre. L'arbre est le symbole de la vie en perpétuelle évolution à travers ses feuilles qui poussent et qui meurent pour repousser au printemps suivant ou encore à travers ses fruits. Parmi les arbres le chêne est l'arbre sacré dans de nombreuses traditions, investi en outre des privilèges de la divinité suprême, symbole de majesté par sa taille, synonyme de force (si en français on dit «fort comme un Turc», en espagnol on dit 
"fuerte como un roble ») et, par son tronc, par ses larges branches, par son feuillage touffu, il est l'emblème de l'hospitalité et l'équivalent d'un temple ${ }^{11}$.

Arbre sacré : Gernikako arbola da bedeinkatua

Euskaldunen artean guztiz maitatua

Adoratzen zaitugu, arbola saindua.

Les termes religieux s'accumulent: bedeinkatua, adoratzen, saindua, la sacralisation est affirmée dès cette première strophe.

Arbre de vie : en un seul vers tout est dit :

etnan ta zabal zazu munduan frutua

Cette formule, à la fois injonction et souhait, évoque d'une manière fort heureuse l'apport que les Basques peuvent faire à l'universel.

L'arbre qui plonge ses racines dans la terre et déploie ses branches et son feuillage vers le ciel établit les rapports entre les univers tellurique et céleste. Ainsi sa naissance se perd dans la nuit des temps, rappelée par Iparraguirre par le nombre mila, mais, par essence, le chêne est divin ce qui est parfaitement souligné dans les deux premiers vers de la seconde strophe :

Mila urte inguru da esaten dutela

Jainkoakjarri zuela Gernikan arbola

Soudain, le doute surgit : l'arbre de vie symbolise la vie même du peuple basque. De sa durée de vie dépend l'existence terrestre des Basques tellement l'un (le chêne) est mythiquement lié à l'autre (le peuple basque). Cette identification est à l'origine même de la douloureuse interrogation, implicite dans l'emploi de l'hypothétique bazera

Zaude, bada, zutikan, orain da denbora

eroritzen bazera arras galdu gera.

2 L'éventualité de la chute du chêne sacré n'a fait qu'effleurer l'esprit. La troisième strophe marque la foi en l'avenir du peuple basque, l'assurance de sa survie, même après les terribles épreuves de la première guerre carliste car il ne faut jamais oublier le contexte historique. C'est une sorte d'envol, de rassemblement des quatre provinces, celles justement qui ont souffert de la guerre, autour de la Junte de Biscaye, celle-là même qui a en charge l'arbre sacré afin que les Basques (euskaldun jendea) puissent vivre en paix.

Etzera eroriko arbola maitea

baldin portatzen bada Bizkaiko Juntea.

Laurok artuko degu zurekin partea

pakean bizi dedin euskaldun jendea.

Enfin le quatrième couplet est celui de la prière. Le chêne, planté par Dieu, donc d'essence divine, ne peut mourir. Mais pour cela il convient d'en faire la demande à Dieu, de se mettre dans les conditions de la prière : belauniko (à genoux), c'est à dire dans l'attitude humble du chrétien qui sollicite la puissance divine. À partir du moment où la prière sera sincère et fervente (biotzetikan) elle sera exaucée; l'arbre vivra éternellement (betiko).

Betiko bizi dedin Jaunari eskatzeko

jarri gaitezen denok laster belauniko.

Eta biotzetikan eskatu ezkero,

arbola biziko da orain eta gero. 
Ainsi se termine ce premier mouvement du Gernikako arbola que l'on peut résumer en la sacralisation du chêne, son identification avec le peuple basque et la foi en sa survie, tout ceci en une langue simple et vigoureuse mise à la portée de tous et, en conséquence, capable d'enflammer tout un auditoire.

Le deuxième mouvement (strophes IV à VIII) est plus politique car il fait allusion à la guerre carliste et surtout à ses conséquences. Ce n'est ni par la foudre, ni de vieillesse que l'arbre de Guernica sera abattu. D'autres forces cherchent ce résultat. Même si elles ne sont pas nommément désignées, tous les Basques mettent un nom derrière le " on » de l'anonymat (dutela) que le basque traduit par le pluriel de troisième personne, cellelà même qui n'est ni toi, ni moi, ni nous, ni vous mais les autres, ceux qui sont en dehors de la communauté basque, affirmée elle par le denok (nous tous) que le démonstratif de proximité ok lie encore plus vigoureusement.

Arbola botatzea dutela pentsatu

Euskalerri guztian denok badakigu.

En face du complot il convient de réagir : Ea (Allons), à la manière de «Allons enfants de la patrie ", hymne que J. M. Iparraguirre connaissait fort bien et dont il mesurait l'allant. C'est l'appel à un élan patriotique destiné à garder l'arbre debout.

Ea, bada jendea, denbora orain degu,

erori gabetanik eduki biagu.

Après cet appel à la mobilisation populaire le poète (strophe VI) s'adresse à l'arbre. $C^{\prime}$ 'est là une technique fort connue de la poésie populaire basque qu'Iparraguirre utilise. Le dialogue est intéressant car il donne un autre rythme au poème, quelque peu différent du premier mouvement. La strophe commence par une louange à l'arbre dont les deux qualités sont mises en valeur : l'éternelle jeunesse ainsi que la pureté, qualités qui divinisent l'arbre.

Beti egongo zera udaberrikoa

lore antziñetako mantxa gabekoa.

Vient ensuite la supplique : Erruki zaitez (Prenez pitié) et la prière de demande du fruit répandu dans les plus brefs délais.

Erruki zaitez, bada, biotz gurekoa,

denbora galdu gabe emanik frutua.

Par ce dialogue l'arbre est bien le lien qui est établi entre la terre et le ciel, entre le peuple basque dont le poète est l'interprète et la divinité.

La réponse de l'arbre (strophe VII) est celle de l'engagement, très liée à l'actualité : un conseil de prudence en premier (kontuz bizitzeko) et la nécessité d'une prière fervente à Dieu qui se résume dans la deuxième partie de la strophe: le refus de la guerre, le besoin d'une paix éternelle basée sur les lois justes et aimées (gure lege zuzenak).

Arbolak erantzun du kontuz bizitzeko

eta biotzetikan Jaunari eskatzeko.

Gerrarik nai ez degupakea betiko,

gure lege zuzenak emen maitatzeko.

L'allusion aux fueros est à peine voilée. Certes, le mot n'est pas prononcé, aucun des interprètes de la chanson, aucun des lecteurs, ne s'y trompe; l'engagement est clairement politique en faveur des fueros. En résumé, le slogan carliste «Dios y los fueros» est implicite qui sera repris plus tard par Sabin Arana Goiri avec son «Jaungoikoa eta lagi zarra». 

Si la première souhaitait la survie du chêne, la seconde manifeste l'aspiration à une paix durable (orain eta beti) sur une terre basque stable, solide et implore le Dieu du ciel qu'il bénisse - et donc prenne sous sa protection -l'Euskal Herri, ce pays des Basques.

Erregutu deiogun Jaungoiko Jaunari

pake emateko orain eta beti

bai eta indarra ere zerorren lurrari

eta bendezioa Euskalerriari. accord avec lui. strophe VI :

Il ne fait aucun doute que les huit premières strophes du Gernikako arbola forment un ensemble cohérent. En effet, après l'exaltation de l'arbre sacré dont la survie s'identifie à la permanence du peuple basque, le second mouvement, après avoir évoqué les dangers qui guettent, engage les Basques à vivre dans la paix grâce à leurs lois ancestrales préservées, les fueros tant aimés, sur un territoire béni par le ciel.

Qu'apporte de nouveau le troisième mouvement? (strophes IX-XII). Pas grand-chose. Ne serait-il pas un ajout ultérieur? C'est fort possible, d'autant qu'il commence d'une manière curieuse :

Orain kanta ditzagun lau bat bertso berri.

C'est, en effet, la manière traditionnelle de commencer une chanson, formule qui, placée au cœur-même d'un poème tel que le Gernikako arbola, ne cesse d'étonner. Le témoignage de José Manterola plaide en faveur de l'ajout quand il écrit :

«Iparraguirre que ha tenido siempre una facilidad asombrosa para improvisar en lengua euskara, introducía en este canto ( Gernikako arbolaC) como en casi todos los suyos, variantes más o menos notables cada vez que cantaba ${ }^{12}$."

À ce sujet José Manterola va jusqu'à condamner l'ajout de ces quatre strophes qui vont même jusqu'à porter préjudice à l'unité du poème, ce en quoi je suis totalement en

«Chaho, Michel, Trueba y otros escritores sólo ofrecen cuatro únicas estrofas... la edición hecha por el señor Santesteban consta a su vez hasta de doce estrofas.

Por mi parte he aceptado esta última versión, que es la más conocida, pero... suprimiendo sus cuatro últimas estrofas que nada añaden de esencial a la composición y que en cambio perjudican, en mi sentir, de una manera notable a su robustez y vigor, haciéndola un tanto pesada y monótona ${ }^{13}$. "

En réalité J. M. Iparraguirre fait entrer en scène les trois provinces, le fameux Irurak bat $\mathrm{du}$ XvIII ${ }^{\mathrm{e}}$ siècle, qui affirment leur adhésion sans réserve. Mais cela ne va-t-il pas en contradiction avec l'idéal d'Iparraguirre qui est de rassembler les quatre provinces, celles-là même qui s'étaient illustrées au cours de la guerre carliste et auxquelles il fait allusion dans le premier mouvement (strophe III).

Laurok artuko degu zurekin partea

pakean bizi dedin euskaldun jendea.

À la strophe XI l'arbre affirme son éternelle jeunesse mais l'allusion existait déjà à la

Beti egongo zera udaberrikoa

et se présente comme le défenseur en face des ennemis. À vrai dire le terme etsaiak n'avait pas été utilisé dans le poème. Il l'est dans cette strophe. L'utilisation de ce terme traduit-elle une époque ultérieure, celle du départ en exil par exemple?

Enfin la douzième strophe nous donnera, peut-être, la clef de l'énigme. Comme les autres mouvements, ce troisième se termine par une prière. Mais, contrairement aux 
deux premiers où le poète s'adresse à Dieu, il s'adresse cette fois-ci à la Vierge. Certes, la dévotion mariale a toujours été très forte en Espagne et au Pays Basque où l'on ne compte plus les sanctuaires qui lui sont dédiés, mais l'année 1854 est une année spéciale dans la chrétienté où a été proclamé par le pape Pie IX le dogme de l'Immaculée Conception dont la fête se célèbre le 8 décembre, jour toujours férié dans la péninsule ibérique. Le culte mariai qui s'en est trouvé renforcé (Lourdes, 1858) n'incite-t-il pas Iparraguirre entre 1855 et 1858, date de son départ en Amérique, à solliciter l'intercession de la Vierge, la Reine du ciel :

Begiratu gaitzazu zeruko erregina.

en même temps qu'il complétait par ce troisième mouvement son Gernikako arbola. Telle est l'hypothèse que je soumets à la critique : ce troisième mouvement qui s'est voulu parallèle aux deux premiers n'est qu'un ajout tardif.

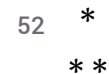

53 Chanté pour la première fois à Madrid en 1853, le Gernikako arbola est propagé par la suite au Pays Basque par son auteur lui-même, éternel vagabond, allant de ville en ville, de bourgade en bourgade, muni de sa guitare afin de gagner sa vie. Il rencontre partout un succès extraordinaire avec sa nouvelle chanson. La preuve nous en est donnée par l'événement qui s'est produit en 1854, au sanctuaire de los Santos Antonios de Urkiola :

« No queremos dejar de mencionar el memorable acontecimiento que tuvo lugar en la campa de este Santuario el año 1854 en que el inmortal José Maria Iparraguirre entonó por primera vez en suelo vascongado, su patriótico canto «Guernica-ko Arbola » en presencia de más de seis mil hombres, que al pronunciar las palabras «adoratzen zaitugu Arbola santua » espontáneamente descubrieron sus cabezas e hincaron sus rodillas en el suelo ; cuyo suceso halló eco en el Salón del Congreso de Diputados de Madrid según atestiguan los discursos de Don Pedro de Egaña, Diputado a la sazón por Alava sirviendo desde entonces el armonioso y viril himno como el más apropiado para despertar amor a las instituciones tradicionales del pueblo euskaldun ${ }^{14}$.»

Le gouvernement espagnol ne s'y est pas trompé; il prend rapidement une sanction d'exil à l'encontre de J.M. Iparraguirre qui repart pour une certaine période sur les routes qui le mènent à Santander, aux Asturies, en Galice, au Portugal, en Andalousie. Son retour au pays lui permettra de rencontrer une jeune fille, sa future femme avec laquelle il s'embarquera à Bayonne en août 1858 à destination du Rio de la Plata. Une nouvelle page est tournée.

L'homme vit en Amérique, mais la chanson demeure qui ne cesse de se répandre à travers tout le Pays Basque. Elle passe la frontière et elle est publiée par FrancisqueMichel en 1857. Mais, en réalité, Francisque-Michel reconnaît qu'il l'a trouvée dans le tome V de l'Histoire des Pyrénées de Cénac Moncaut, ouvrage paru en 1855.

«Le chant national suivant, dit M. Cénac Moncaut, qui l'a reproduit, joint à une certaine valeur poétique l'intérêt historique que doit naturellement exciter l'arbre colossal et séculaire auquel il est dédié, et sous lequel la junte d'Alava ${ }^{15}$ tint ses réunions pendant plusieurs siècles, comme le bilzaar (sic) se réunissait sous le chêne d'Ustaritz. »

Après cette citation, Francisque-Michel poursuit :

« Nous le publions d'après un placard qui renferme une autre chanson patriotique et qui se termine ainsi: Tolosan, Andres Gorosabelen echean 1856. Ces deux morceaux sont anonymes; mais s'il faut en croire ce qui nous a été dit, l'auteur de celui que nous donnons serait un certain Ipharraguirre ${ }^{16}$. » 
57 Pas plus que le gouvernement espagnol, Francisque-Michel ne se trompe sur l'exacte valeur du Gernikako arbola qu'il classe parmi les chansons politiques en même temps que celle de Muñagorri, elle aussi en faveur des fueros. Il aurait eu une raison supplémentaire de la classer dans les chansons politiques s'il avait connu les strophes suivantes - du moins les quatre suivantes - dont on est sûr qu'il les aurait publiées s'il les avait recueillies. Mieux que cela, alors que Cénac Moncaut parle de «chant national ", Francisque-Michel dans son commentaire n'hésite pas à qualifier le Gernikako arbola de "chant patriotique ». Il est intéressant d'apprendre aussi que la diffusion se fait par «placard» ou feuille volante, c'est-à-dire d'une manière traditionnelle. Était-ce la première correspondant à ce poème qui était publiée en Pays Basque?

Ce « certain Ipharraguirre » (avec orthographe utilisant le phonème labial aspiré alors qu'il n'existe pas dans les provinces du Sud) n'est pas tout à fait un inconnu dans les provinces du Nord. En effet, il a séjourné à Bayonne, connu Augustin Chaho dont on sait l'engagement carliste et a été invité au séminaire de Larressore en 1845-1846 par le Supérieur Haramboure où, selon le témoignage de Gratien Adéma, il avait chanté quelques zortzicos. On sait par Gratien Adéma que, parmi ces zortzicos, il avait chanté le fameux "Gitarra zartxo bat da neretzat laguna " où il narre ses errances à travers l'Europe et se définit comme artiste basque ${ }^{17}$. En effet, dès 1856 , on trouve dans les poèmes envoyés aux Jeux floraux d'Urrugne une chanson d'un certain Paul Louis sur le thème imposé cette année-là de la fête au village. La chanson a pour titre Herrietako bestak et elle peut être chantée sur deux airs différents : "Donostiara noha, Josepa, adio! edo Gitarra txartxo bat nerekin laguna.» Deux remarques: l'incipit du timbre n'est qu'approximativement cité ce qui signifie qu'il a été entendu, mais pas bien retenu, et, d'autre part, on ignore qui est ce Paul Louis. Ne serait-ce pas le pseudonyme d'un prêtre basque? L'homme est en tous cas un lettré qui, avant d'indiquer le timbre, écrit en latin «Nihil operis facietis in eo " et fait allusion dans le texte basque aux Grecs, à l'Olympe, à Pindare. En outre, ses quatrains n'obéissent pas totalement à la norme traditionnelle qui veut qu'ils soient monorimes car ils sont faits d'une double rime plate à la manière de la prosodie française. L'auteur ne serait-il pas un jeune prêtre, séminariste en 1845-1846 et qui, à Larressore, aurait entendu chanter Iparraguirre?

Le timbre du Gernikako arbola a donné lieu à de nombreuses discussions, certains l'attribuant à Altuna, le pianiste qui accompagnait J. M. Iparraguirre à Madrid au café San Luis, alors que la majorité penche pour Iparraguirre lui-même, dont le savoir musical est connu.

60 En me référant au témoignage de mon collègue d'Euskaltzaindia, José Antonio Arana Martija, musicologue distingué, l'air du Gernikako arbola proviendrait d'une danse recensée dès 1711 à Garay (Biscaye) et connue sous le nom de Dantzari dantza ou danse du danseur. Selon Arana Martija cet air aurait pu être entendu aussi bien par Altuna, Durangues de naissance que par Iparraguirre lorsqu'il était à la Garde d'Honneur du Roi Carlos V qui a séjourné à Durango durant la première guerre carliste ${ }^{18}$. Tout porte à croire que, l'ayant repris de la tradition orale, Iparraguirre ait adapté l'air pour en faire l'hymne connu. De nombreux musiciens n'ont pas agi autrement.

61 Laissant de côté l'origine, il faut reconnaître que le timbre du Gernikako arbola a eu un grand retentissement en Pays Basque, par sa facture sans doute, mais certainement tout autant sinon plus par le poème qu'il accompagne. C'est, en effet, dans une période inquiétante pour le Pays Basque et son statut à la fois juridique et politique, un cri en 
faveur des fueros et, implicitement, de la liberté. D'où l'accueil enthousiaste dont le témoignage le plus flagrant est le fameux rassemblement d'Urkiola et la manière dont a été reçu le message.

En 1858, c'est-à-dire à peine cinq ans plus tard, un anonyme présente aux Jeux floraux d'Urrugne une chanson qui adopte le timbre du Gernikako arbola et s'intitule: 0 Gernikako haritz. Airea: Gernikako zuhaitza. ${ }^{19}$ On pourrait imaginer que derrière cet anonymat se cache un poète du Pays Basque Sud. Il n'en est rien car la langue et l'orthographe sont labourdines. D'autre part, l'auteur néglige la monorimie au profit de deux rimes plates à la française dans les huit quatrains $(a-a-b-b)$. Il célèbre la naissance du prince impérial, fils de Napoléon III et d'Eugénie de Montijo n'oubliant pas de signaler que l'impératrice est d'ascendance ibère (basque dans son esprit) et s'adressant à l'Empereur il écrit :

Barkha ezaguzuke gure ausartzia,

Ibero alaba da zure Eugenia.

Tout comme le Gernikako arbola, le poème est composé de quatrains, mais on ne sait de quelle manière l'auteur le chantait. En effet, le texte donné par Francisque-Michel en 1857, tiré d'une feuille volante publiée à Tolosa l'année précédente est présenté à la manière du Sud comme un zortziko : huit vers dont seuls les vers pairs riment, les quatre derniers devant être bissés. On a donc affaire, du moins pour le timbre, à un passage musical nouveau pour le bis final, ce qui pourrait correspondre à un quatrain quand les paroles sont répétées ou de quatre vers nouveaux si l'auteur propose de nouvelles paroles. Ainsi, de huit vers courts on peut passer à douze vers et, pour les Basques du Nord, d'un quatrain à un sizain.

Il faut attendre 1868 pour retrouver dans les Jeux floraux le timbre du Gernikako arbola. C'est un poème dédié à l'Impératrice, Enperatrizari, composé par Oxalde qui fut douanier puis facteur, improvisateur renommé, qui, grâce à cette chanson, obtient le premier prix à Sare où Antoine d'Abbadie a fixé depuis quelques années le lieu du concours. En neuf strophes, un soldat raconte la campagne d'Italie : le pont de Magenta, la bataille de Solférino, l'aide des troupes françaises au pape face aux Italiens qui veulent s'emparer des Etats pontificaux, son retour au pays où l'Impératrice passe chaque année ses vacances.

Dans le dernier couplet il exhorte les jeunes à bien servir l'Empereur et à suivre son exemple.

Bide beretik habil, hi ere gaztea! (berriz)

Curieusement, les strophes ne sont pas des quatrains mais des quintils monorimes à la manière traditionnelle qui, par le redoublement du dernier vers, nous conduisent vers le sizain commandé par le timbre.

Ayant obtenu le premier prix, le poème doit être chanté en public afin d'honorer son auteur. C'est donc l'air du Gernikako arbola qui sera entonné sur la place de Sare. À ce sujet la lettre du capitaine Duvoisin à Antoine d'Abbadie ne manque pas d'intérêt :

Bayonne 25 août 1868

Monsieur,

La pièce envoyée au concours de poésie basque par le $S^{\prime}$ Oxalde, facteur rural à Briscous, nous a quelque peu embarrassés et nous avons pris conseil avant de lui adjuger le prix. Il n'y avait pas de reproche à lui adresser ni sur la pureté, ni sur la correction du langage, non plus que sur le fond des pensées. Ce morceau tient un peu trop du récitatif; on y trouve cependant de belles strophes qu'on chercherait en vain dans plusieurs des pièces antérieurement couronnées. Nous avons cru qu'il 
y aurait injustice à refuser le prix à une composition qui leur est supérieure. En tenant ainsi la main à ce que le niveau poétique s'élève de plus en plus, le résultat que vous cherchez sera, je crois, atteint. L'air choisi est très musical Gernikako arbola, mais peu connu ici. Il était à craindre que les chanteurs de Sare ne sussent pas s'en tirer. L'auteur a donc confié l'exécution à un jeune homme de Briscous, doué d'une bonne voix; et particularité singulière pour chanter le retour d'un soldat de Solférino, l'acteur porte une belle balafre au travers de la figure.

Je vous prie.... ${ }^{20}$ 
montagne mythique, sujet de nombreuses chansons, encore plus chère au coeur d'un Saratar comme l'est Agustin Etcheverry.

En 1879 Antoine d'Abbadie décide que les concours auront lieu en alternance dans les sept provinces basques, une année au Sud, l'autre au Nord. Désormais les poètes biscayens et guipuzcoans qui, timidement, avaient commencé à se présenter, prendront nettement l'avantage avec des thèmes nouveaux, en particulier celui de la langue avec les Arrese-Beitia, Ramón Artola, Antonio Arzak, Klaudio Otaegi etc. qui se servent beaucoup plus du hamarreko haundi au vers rythmé en $5+5+8$, totalement différent du zortziko txiki d'Iparraguirre quelque peu délaissé.

En 1884, à Sare où a lieu le concours, un témoignage sur le Gernikako arbola nous est apporté par le maire de Sare Elissague qui décrit la fête à Antoine d'Abbadie qui n'a pu la présider comme d'habitude. Cette année-là c'est Pierre Dibarrart qui l'a emporté avec Usoa saretan atzemana (La palombe prise aux filets) et de la longue missive du maire de Sare du 13 septembre 1884 est extrait ce passage intéressant :

« Après dîner et à 3 heures, en présence d'une foule immense la chanson couronnée (Usoa saretan atzemana) a été chantée par le chantre d'Ustaritz. Suivie de l'Arbol de Guernica magistralement joué par la musique de Hendaye, elle a excité un enthousiasme indescriptible. Connaissant votre ardent patriotisme, il nous a été particulièrement pénible de vous savoir absent au moment d'une manifestation basque si belle et si spontanée. Lorsqu'enfin la foule cessa ses hourrahs frénétiques et ses applaudissements, les improvisateurs commencèrent ${ }^{27}$. "

76 Ainsi le poème primé est chanté et, immédiatement après, un ensemble musical de Hendaye interprète le Gernikako arbola, donc sans paroles, instauré comme véritable hymne destiné à honorer le lauréat et son œuvre. C'est ensuite l'explosion populaire qui porte tout autant sinon plus sur l'hymne que sur la poésie. Il ne fait aucun doute que le Gernikako arbola est reconnu comme hymne basque par la foule et le patriotisme d'Antoine d'Abbadie en aurait été comblé. Il est possible que le Gernikako arbola ait déjà eu cet honneur, nous en avons ici un témoignage daté.

En 1891 à Yurreta (Biscaye) c'est à nouveau Dibarrart qui chante Euskaldunen loriak (Les gloires des Basques), treize quatrains sur le rythme du zortziko txiki $(7+6)$ et sur l'air de Adio Euskal Herria ou Gernikako arbola ${ }^{28}$. Trois ans plus tard Dibarrart présentera ce même poème, mais remanié, aux fêtes basques de Saint-Jean-de-Luz organisées par la municipalité et son maire le $\mathrm{D}^{\mathrm{r}}$ Gratien Albert Goyheneche, sous la présidence conjointe de la Reine Nathalie de Serbie et d'Antoine d'Abbadie.

78 À ce même concours de Yurreta un anonyme envoie Nere mendiyak (Mes montagnes), toujours en quatrains ou plutôt zortzikos car le poète est manifestement quelqu'un du Pays Basque Sud ${ }^{29}$. Hommage aux montagnes basques autour desquelles on entend la langue naturelle, l'euskara.

79 Au total dans les Jeux floraux de cette deuxième moitié du xix siècle, le timbre du Gernikako arbola apparaît dix fois, timbre le plus employé, ce qui prouve à quel point il est apprécié. Il est suivi par Hau da ikazketako mandoaren traza, ou chant du muletier au rythme allègre, utilisé surtout par sept fois dans la période 1853-1865.

Il est certain que le timbre du Gernikako arbola a été utilisé en dehors des Jeux floraux. Je n'en veux pour preuve que ce qu'en dit José Antonio Arana Martija :

«Años más tarde cuando Gratien Adema «Zaldubi» utilizó la mclodía del Gernikako Arbola como himno al Papa en su peregrinatión ( 1870) compuso una estrofa de diez versos (hamarreko txikia) con cinco rimas : hori, orori, Erromari, Sainduari, $\operatorname{amar}^{30}$. " 
81

Plusieurs années plus tard en 1877, le même Zaldubi créa une chanson pour un pèlerinage en Terre sainte qui se trouve dans l'opuscule intitulé Escualdun Pelegrinaren bidaltzailea (Le Guide du pèlerin basque) et dans le même article José Antonio Arana Martija rappelle que Resurrección Maria de Azkue, premier président d'Euskaltzaindia, entendit dans une église de Gijón chanter le cantique

Venid y vamos todos

con flores a Maria...

sur l'air du Gernikako arbola ${ }^{31}$. Apport manifeste du profane au sacré.

D'autre part, mon ami et collègue à Euskaltzaindia Pierre Charritton me fait savoir que Mendiague, poète haspandar, exilé en Amérique latine, a, par deux fois, utilisé le timbre Gernikako arbola, en 1890 dans EuskaldunakBuenos Aires-etako Esposizionean (Les Basques à l'exposition de Buenos Aires) composé de sept quatrains ${ }^{32}$ et en 1894 dans Eskualdun baten kontseiluak Montevideoko Presidentari (Les conseils d'un Basque au président de Montevideo), cette fois-ci en vingt quintils adressés à Idiarte-Borda, président de la République d'Uruguay, d'ascendance basque ${ }^{33}$.

$84 *$

$* *$

On a déjà vu qu'à Sare, en 1884, le Gernikako arbola, joué par un groupe musical hendayais avait soulevé l'enthousiasme de la foule. Outre les Jeux floraux, les fêtes basques vont se multiplier, un particulier à Saint-Jean-de-Luz sous l'impulsion du maire le $\mathrm{D}^{\mathrm{r}}$ Goyheneche. En 1892, on célèbre Antoine d'Abbadie qui a été élu président de l'Académie des Sciences. Quatre discours, ceux du maire Goyheneche, de M. J.D.J. Sallaberry de Mauléon, de M. de Souhy, maire de Mauléon et de M. Berdeco, avocat à Saint-Palais, ont précédé celui de M. Charles Petit, chargé d'offrir à Antoine d'Abbadie «un makhila d'honneur à pomme d'or avec inscription commémorative». Le journaliste qui relate la cérémonie ajoute :

"Quand il a eu fini, une triple salve d'applaudissements a ébranlé la salle et

l'assistance tout entière, debout, a entonné en chœur notre chant national, cet

admirable hymne d'Iparraguirre si cher aux Basques des deux frontières :

Guernicaco arbola, guziz maitatua!

$\mathrm{Ah}$ ! c'était bien là une fête d'enfants du pays ${ }^{34}$ !»

En 1894, les fêtes basques sont placées sous la présidence d'honneur de Sa Majesté la Reine Nathalie de Serbie et de M. Antoine d'Abbadie, membre de l'Institut. Un nouveau chant patriotique - ainsi est-il désigné - apparaît alors, chant présenté au concours d'Azpeitia l'année précédente et remanié par son auteur Gratien Adéma, «Zaldubi ». Ce chant des fêtes et réunions basques est entraînant. Il est intitulé Gauden gu eskualdun (Demeurons basques) et son refrain prône l'union des sept provinces :

Zazpi Eskual-herriek bat egin dezagun

Guziak bethi bethi gauden gu Eskualdun ${ }^{35}$

87 Toutefois ce chant des fêtes et réunions basques n'est nullement destiné à détrôner le Gernikako arbola qui demeure l'hymne basque créé en cette moitié du XIX siècle. Je n'en veux pour preuve que la fin de l'article que Zaldubi lui-même consacre à ces fêtes dans l'Eskualduna:

«Zein den eder orduan, guziek, chutik eta buru has, elgarrekin kanta dezagun

Gernikako arbolaren kanta sustagarria, eta bertze kanta laphurtarrenaren oihu hau :

Zazpi Eskual-herriek bat egin dezagun

Guziak bethi bethi gauden gu Eskualdun ${ }^{36}$." 
88

Les deux chants vont de pair ; ils marquent deux époques différentes, celles du milieu $\mathrm{du}$ XIX ${ }^{\mathrm{e}}$ siècle pour le Gernikako arbola, celle de la fin du siècle pour le Gauden gu Eskualdun, mais l'auteur du second, comme on l'a vu, reconnaît la prééminence du premier. Pendant plus d'un siècle il a été, par excellence, l'hymne de tous les Basques. Il est regrettable qu'il ait été écarté par le Parlement de la Communauté autonome basque, partie importante certes, mais partie de l'ensemble du Pays Basque.

\section{NOTES}

1. M. Lekuona, Literatura oral euskérica, Donostia, 1936, p. 52.

2. Kantu, kanta, khantore, Bayonne, 1967, p. 67.

3. José Mendiague, Euskaldun kantak, Buenos Aires, 1900, p. 54.

4. R.P. Onaindia, Mila euskal olerki eder, Larrea-Amorebieta, 1954, p. 536.

5. Messager de Bayonne, $\mathrm{n}^{\circ} 296$, jeudi 9 juin 1853.

6. M. Lekuona, Literatura oral euskérica, Donostia, 1936, p. 133.

7. M. Lekuona, Literatura oral euskérica, Donostia, 1936, p. 134.

8. Federico Zabala, "Iparraguirre y su entorno Los fueros", in Iparraguirre, Jagon 2, Euskaltzaindia, Bilbao, 1987, p. 92.

9. Federico Zabala, "Iparraguirre y su entorno Los fueros", in Iparraguirre, Jagon 2, Euskaltzaindia, Bilbao, 1987, p. 92.

10. Cité par J.M. Lekuona, «Iparragirre eta bertsolaritza", in Iparraguirre, Jagon 2, Euskaltzaindia, Bilbao, 1987, p. 118.

11. Jean Chevalier, Alain Gheerbrant, Dictionnaire des symboles, Robert Laffont, Paris, 1982, p. 221.

12. J. Manterola, Cancionero vasco, $2 \mathrm{~s}$, III t, Juan Osés, Donostia, 1877, p. 79.

13. J. Manterola, op. cit, p. 79-80. J'ai tiré ces deux citations de l'article de J.M. Lekuona (voir note 10), p. 112 et 118.

14. Benito de Vizcarra y Arana, Reseña histórica del multisecular Santuario de los Santos Antonios de Urquiola, Montepio Diocesano, Vitoria, 1932, p. 23-24.

15. Erreur manifeste de Cénac Moncaut. Gernika est en Biscaye.

16. Francisque-Michel, Le Pays Basque, sa population, sa langue, ses mœurs, sa littérature et sa musique, 1857, p. 262-263.

17. Charles Bernadou, Azpeitia, Les Fêtes euskariennes de 1893, Lasserre, Bayonne, 1894, p. 72-73 Adéma ajoute que, chauffé par la salle, Iparraguirre s'est lancé dans des improvisations. «Parmi les nombreuses strophes que chanta ce soir-là Iparraguirre il y avait certainement quelques vers du Gernikako arbola. Ce premier jet du poète avait-il déjà les neuf premières strophes? On sait qu'il y en a douze aujourd'hui. » Témoignage bien flou - 48 ans se sont écoulés - et le «certainement " m'apparaît surtout exprimer le doute. D'autre part pourquoi neuf strophes? Là aussi on reste dans le flou.

18. J.A. Arana Martija, «En torno a la musica y letra del Gernikako arbola », Txistulari, Bilbao, ${ }^{\circ}$ 47, Juillet-août-septembre 1966, p. 35.

19. P. Urkizu, Anton Abbadiaren koplarien guduak, Donostia, Eusko Ikaskuntza Euskaltzaindia, 1998, p. 121.

20. Bibliothèque Nationale, Paris, Section des manuscrits, NAF 21747, folio 196.

21. P. Urkizu, op. cit, p. 241-242.

22. P. Urkizu, op. cit, p. 250. 
23. P. Urkizu, op. cit, p. 264.

24. P. Urkizu, op. cit, p. 265.

25. P. Urkizu, op. cit, p. 272-273.

26. P. Urkizu, op. cit, p. 288-289.

27. Bibliothèque Nationale, Paris, Section des manuscrits, NAF 21747, folio 274-275.

28. P. Urkizu, op. cit, p. 376-377.

29. P. Urkizu, op. cit, p. 377.

30. J.A. Arana Martija, "Gernikako arbola », in José Maria Iparraguirre, erro-urratsak, Raízy viento, Keinu, 1999, p. 107.

31. J.A. Arana Martija, "Iparragirre eta Bizkaia " (Iparraguirre et la Biscaye) in Iparraguirre, Jagon 2, Euskaltzaindia, Bilbao, 1987, p. 294-295.

32. José Mendiague, Eskualdun kantuak (Chants basques), Buenos Aires, 1900, p. 152-153.

33. José Mendiague, op. cit, p. 36-39.

34. Eskualduna, Bayonne, 16 septembre 1892.

35. Eskualduna, Bayonne, 24 août 1894.

36. Eskualduna, Bayonne, 7 septembre 1894.

INDEX

Mots-clés : bertsulari, bertsularisme, chanson, fors (droit), symbole

Index chronologique : 19e siècle

Thèmes : linguistique, philologie

Index géographique : Biscaye, Guernica

\section{AUTEUR}

\section{JEAN HARITSCHELHAR}

Professeur émérite de l'Université Michel de Montaigne - Bordeaux III, UMR 5478

baionaordez@euskaltzaindia.net 